\title{
Estudo de Validade do DFH como Medida de Desenvolvimento Cognitivo Infantil
}

\author{
The Draw-a-Person Test as a Valid Measure of Children's Cognitive Development
}

\author{
Denise Ruschel Bandeira*, Angelo Costa \& Adriane Arteche** \\ Universidade Federal do Rio Grande do Sul
}

\begin{abstract}
Resumo
O Teste do Desenho da Figura Humana (DFH) é amplamente utilizado como uma técnica de avaliação do desenvolvimento cognitivo, porém, o reconhecimento da validade deste instrumento ainda hoje não está completo. No Brasil, o Sistema Wechsler de avaliação cognitiva do DFH já foi aprovado pelo Conselho Federal de Psicologia. No entanto, considerando a necessidade de periodicamente se reavaliar o instrumento, o objetivo deste estudo foi avaliar a validade convergente e concorrente do DFH-Sistema Cognitivo de Wechsler. Para validade convergente foi utilizado o teste das Matrizes Coloridas Progressivas de Raven, validado nacional e internacionalmente. Para validade concorrente, utilizou-se uma escala de desempenho escolar. Participaram 90 crianças, de 6 a 12 anos $(M=8,99$ e $D P=1,79), 37$ meninas e 53 meninos, oriundas de escolas públicas (préescola a $6^{a}$ série), que não realizavam atendimento psicológico e não tinham problemas de aprendizagem. Os resultados apontam a existência de correlações significativas moderadas entre o DFH, o Raven e a escala de desempenho escolar, confirmando que o DFH-Sistema Wechsler é válido como medida do desenvolvimento cognitivo. Sugere-se, então, que o DFH possa ser incluído em baterias de avaliação de crianças ou em triagens para futuras avaliações mais complexas.

Palavras-chave: Desenho da Figura Humana; validade convergente e concorrente; inteligência.
\end{abstract}

\begin{abstract}
Draw-a-Person Test (DAP) is widely used to assess cognitive development, although, the recognition of the validity of this instrument is not complete. In Brazil, the Wechsler system of evaluation of DAP has already been approved by the Federal Psychology Board. However, considering the need to periodically re-evaluate an instrument, this study attempted to test both convergent and concurrent validity of the DAP - Cognitive System according to Wechsler. Thus, Raven's Progressive Matrices, a nationally and internationally validated instrument, was used to analyze convergent validity and a school performance scale was used to analyze concurrent validity. Ninety children from 6 to 12 years old $(M=8,99$ and $S D=1,79), 37$ girls and 53 boys, from public schools (kindergarten to $6^{\text {th }}$ grade), who did not engage in psychological treatment and did not have learning disabilities participated in this study. Results showed moderate positive correlations among the three instruments, confirming that DAP scored via Wechsler' system is a valid measure of cognitive development. Suggestions are made in the sense of including DAP in assessment batteries or using it as a brief assessment.

Keywords: Draw-a-Person Test; convergent and concurrent validity; intelligence.
\end{abstract}

Na história da humanidade verifica-se que o desenho é uma das formas de comunicação mais utilizadas pelo homem e antecedeu à escrita, indicando assim que a comunicação por meio de desenhos é uma forma de linguagem básica e universal (Wechsler, 1996/2003). No meio acadêmico, entretanto, este passou a ser percebido não apenas como uma forma de comunicação, mas também como técnica de avaliação psicológica.

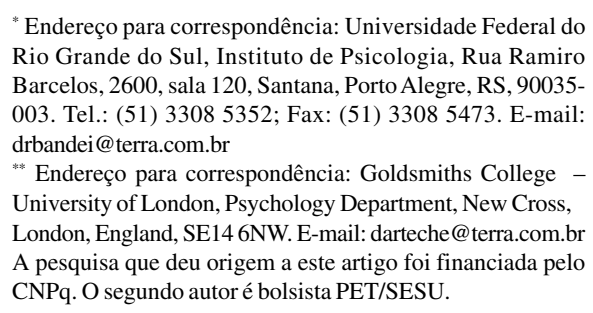

*Endereço para correspondência: Universidade Federal do Rio Grande do Sul, Instituto de Psicologia, Rua Ramiro Barcelos, 2600, sala 120, Santana, Porto Alegre, RS, 90035 003. Tel.: (51) 3308 5352; Fax: (51) 3308 5473. E-mail: drbandei@terra.com.br

** Endereço para correspondência: Goldsmiths College University of London, Psychology Department, New Cross, London, England, SE146NW. E-mail: darteche@terra.com.br A pesquisa que deu origem a este artigo foi financiada pelo CNPq. O segundo autor é bolsista PET/SESU.

O Desenho da Figura Humana (DFH) como técnica de avaliação psicológica possui diversos sistemas de interpretação, tanto de cunho evolutivo quanto emocional. Para avaliação de aspectos evolutivos, destacam-se aqueles sistemas que compreendem o desenho como medida de avaliação do desenvolvimento cognitivo infantil, como o de Goodenough (Alves, 1981), o de Koppitz - Indicadores Desenvolvimentais (Koppitz, 1984), o de Naglieri (1988) e, no Brasil, o de Wechsler (1996/2003) e o de Sisto (2006).

Enquanto medida de avaliação dos aspectos cognitivos o desenho é entendido como expressão de aspectos desenvolvimentais existindo um ciclo infantil típico que pode ser observado a partir da produção gráfica. Segundo Abou-Jamra e Castillos (1987), há uma íntima relação entre o desenho e o desenvolvimento conceitual. Inicialmente as crianças desenham o que sabem e não o que vêem. Com o desenvolvimento, a criança tentará mais e mais re- 
presentar os objetos como os vê, surgindo gradualmente os conceitos de tamanho, proporção, posição relativa das partes, relação espacial e outros. Ainda que existam controvérsias sobre as etapas do desenho e, especialmente, sobre a linearidade do processo de desenvolvimento destas, a maior parte dos autores reconhece estágios típicos neste processo.

Florence Goodenough, em 1926, foi a pioneira na tentativa de sistematizar este pressuposto em um sistema de avaliação dos desenhos infantis (Goodenough, 1974). Em 1963, este trabalho foi revisado e ampliado com a colaboração de Dale Harris e utilizado como indicador de maturidade intelectual e não de inteligência (Cox, 1995). Em 1968, Elisabeth Koppitz iniciou o desenvolvimento de um outro sistema de análise do desenho. A fim de elaborar a lista dos itens desenvolvimentais, a autora selecionou, a partir da proposta de Goodenough e Harris e da sua própria experiência, uma lista de itens considerados de natureza evolutiva (Koppitz, 1984).

No Brasil, Solange Wechsler, baseando-se nos sistemas de Harris, Koppitz e Naglieri (Wechsler, 1996/2003), elaborou um sistema quantitativo de avaliação do desenvolvimento cognitivo a partir do DFH. A primeira edição de seu estudo foi publicada em 1996 na qual foram apresentados os indicadores de validade e precisão da proposta. Posteriormente, em 2000, foram apresentados os estudos nacionais e transculturais que corroboravam os resultados apresentados quatro anos antes e, em 2003, foi publicada a edição revisada e atualizada, com normas referentes a várias regiões brasileiras.

A versão atual proposta por Wechsler (1996/2003) sugere a aplicação de dois desenhos: homem e mulher, que são corrigidos independentemente, mas cujos resultados serão somados a fim de se obter uma medida final. $\mathrm{O}$ desenho da figura feminina é avaliado conforme 17 itens. Já a figura masculina é avaliada conforme 18 itens, também subdivididos. Cada um dos itens é pontuado com um ponto quando presente. Os resultados brutos de cada figura e do total são convertidos em resultados padronizados considerando o sexo da criança que desenhou e a sua faixa etária. A partir do resultado padronizado obtém-se a classificação e o percentil. Além disso, é possível identificar em uma lista de itens desenvolvimentais, aqueles que são esperados, comuns, incomuns e excepcionais para cada faixa etária.

No que diz respeito aos estudos de validade do DFHCognitivo, alguns estudos têm mostrado correlações positivas entre o desenho e testes que avaliam a inteligência (Abell, Horkheimer \& Nguyen, 1998; Parvathi \& Natarajan, 1985; Wechsler, 1996/2003). Embora as referidas pesquisas tenham utilizado diferentes sistemas de levantamento do DFH, todas chegam às mesmas conclusões. Parvathi e Natarajan inclusive chegam a afirmar que a habilidade de desenhar em crianças é muito dependente da sua inteligência abstrata.

Seguindo essa linha, a fim de buscar evidências de validade do seu teste, Wechsler e Schelini (2002), propuseram um estudo correlacionando os resultados do DFH com o
Teste Não-Verbal do Raciocínio Infantil (TNVRI). Foram testadas 103 crianças, com idades entre 9 e 11 anos. Os resultados mostraram que existe relação entre o desenvolvimento cognitivo infantil, quando medido pelo desenho, e o raciocínio analógico, de forma concreta ou abstrata ( $r=0,21$ a 0,27 , conforme o sexo do desenho). Considerando-se que esta correlação não foi bastante forte, pode-se afirmar que os dois testes estão avaliando conceitos diversos, embora tenham sobreposições em relação ao conteúdo medido. Outro motivo pelo qual a correlação pode ter sido mais baixa do que a esperada tem relação com o teste que foi utilizado no estudo de Wechsler (1996/ 2003). Este é um teste que foi desenvolvido recentemente pela equipe do Laboratório de Pesquisa em Avaliação e Medida (LabPam), da Universidade de Brasília (UnB), sobre o qual não foi encontrado estudo de validação nos sistemas de busca nacionais, apesar de ter sido aprovado pelo Conselho Federal de Psicologia (CFP). Conforme Pasquali (2005), a validade e precisão desse instrumento ainda possui caráter exploratório.

Os estudos encontrados mostram que realmente há controvérsias quanto à correlação entre o DFH e outra medida de inteligência. Abell, Wood e Liebman (2001) encontraram correlações mais altas, especialmente entre crianças menores de 11 anos, entre o DFH (avaliado pelos sistemas de Goodenough e Harris e de Naglieri) e o WISC-R e o WISC-III (variando de $r=0,36$ a $r=0,57$ na sub-escala de execução). Contudo, os autores comentam que esses valores explicam uma pequena porção da variância e sugerem que o DFH seja aplicado somente numa bateria com outros testes. Resultados semelhantes foram encontrados por Abell, Horkheimer e Nguyen (1998) em outro estudo com 200 meninos. Kamphaus e Pleiss (1991) sugerem que os indicativos de validade do DFH advêm, essencialmente, de estudos de validade concorrente.

No Brasil, recentemente foi publicado um estudo de correlação entre o DFH - Escala Sisto e o Raven (Marín Rueda $\&$ Sisto, 2006). Os autores compararam os desenhos de 279 crianças de 7 a 10 anos, avaliados conforme o Sistema Sisto (um sistema de levantamento do DFH baseado em Goodenough) com seu desempenho no teste das Matrizes Progressivas Coloridas de Raven. Para análise dos dados, as crianças foram classificadas pelo seu rendimento no Raven e posteriormente comparadas quanto ao número total de itens da escala do DFH. Os resultados mostraram que as crianças são diferenciadas no DFH-Escala Sisto apenas para as idades de oito, nove e 10 anos. Para as menores, sete anos, não foi encontrada diferença.

Como pode ser visto, novos estudos de validação são necessários a fim de aprimorar a técnica e atender à Resolução que o CFP (2003) publicou recentemente acerca da utilização de instrumentos de avaliação psicológica. Ainda que o DFH para avaliação dos aspectos cognitivos, segundo o Sistema de Wechsler (1996/2003), conste como uma técnica válida, é importante que os instrumentos sejam constantemente revalidados, em regiões variadas do país. Além disso, cabe ressaltar que para a região sul do 
Brasil, ainda não foi realizado nenhum estudo de validação do DFH - Sistema Wechsler.

Uma das formas de validação possível para este teste é o estudo de sua relação com testes de inteligência. Nesse tipo de estudo utiliza-se como referência um teste já validado com o qual o teste em questão dever se correlacionar (Pasquali, 2001). O Teste das Matrizes Progressivas Coloridas de Raven é uma das mais utilizadas medidas de raciocínio abstrato, tendo sua validade atestada nacional e internacionalmente (Angelini, Alves, Custódio, Duarte \& Duarte, 1999; Bandeira, Alves, Giacomel \& Lorenzatto, 2004; Costenbader \& Ngari, 2001; Pind, Gunnarsdóttir \& Jóhannesson, 2003; Powers \& Barkan, 1986).

O Teste das Matrizes Progressivas Coloridas de Raven, desenvolvido por John C. Raven, foi padronizado e publicado em 1947. No Brasil, foi publicado pela Casa do Psicólogo em 1988 como um manual abreviado da adaptação brasileira e, em 1991, tornou-se disponível para o mercado o manual completo (Angelini et al., 1999). As Matrizes Progressivas Coloridas de Raven podem ser empregadas com crianças pequenas, pessoas idosas e deficientes mentais, destinando-se à faixa de 5 a 11 anos, no que se refere a crianças.

Considerando os aspectos acima referidos acerca do DFH cognitivo e seu processo de validação, a presente pesquisa visou buscar evidências de validade convergente e concorrente do DFH, correlacionado-o com o Teste Raven em crianças de 6 a 12 anos e uma escala de avaliação de desempenho escolar das crianças, respondida pelas respectivas professoras. Para análise dos desenhos, foi utilizado o Sistema Cognitivo de Wechsler, considerando a carência de estudos brasileiros de validação desse sistema e por ser o mais atual, construído especificamente para a população brasileira.

\section{Método}

\section{Participantes}

Participaram desta pesquisa 90 crianças de nível sócioeconômico baixo ou médio-baixo, com idades entre 6 e 12 anos $(M=8,99$ e $D P=1,79)$ de ambos os sexos, sendo 53 meninos e 37 meninas (Tabela 1). Os participantes fizeram parte do Grupo Controle de um projeto de construção e validação de uma escala infantil de indicadores emocionais do desenho da figura humana (Arteche, 2006), composto por estudantes de escolas públicas que não se encontravam em atendimento psicológico ou pedagógico e que foram avaliados pelas professoras como não tendo dificuldades de aprendizagem e/ou de relacionamento. Os participantes freqüentavam da pré-escola $(7,2 \%)$ até $6^{\mathrm{a}}$ série $(8,2 \%)$, sendo que a maioria freqüentava a $2^{\mathrm{a}}$ e a $4^{\mathrm{a}}$ série (17,5\% cada). Com relação ao nível sócio-econômico, este não foi diretamente avaliado. $\mathrm{O}$ fato das crianças pertencerem a escolas públicas de zonas centrais da cidade indica que seu nível sócio-econômico é de médio a médio-baixo.
Tabela 1

Dados Sóciodemográficos dos Participantes

\begin{tabular}{lcc}
\hline & Meninos $F(\%)$ & Meninas $F(\%)$ \\
\hline Faixa etária & & \\
6 anos & $7(13,2 \%)$ & $4(10,8 \%)$ \\
7 anos & $3(5,7 \%)$ & $5(13,5 \%)$ \\
8 anos & $9(17,0 \%)$ & $7(18,9 \%)$ \\
9 anos & $10(18,9 \%)$ & $9(24,3 \%)$ \\
10 anos & $11(20,8 \%)$ & $5(13,5 \%)$ \\
11 anos & $8(15,1 \%)$ & $3(8,1 \%)$ \\
12 anos & $5(9,4 \%)$ & $4(10,8 \%)$ \\
Escolaridade & & \\
Pré-escola & $4(7,5 \%) 7$ & $4(10,8 \%)$ \\
$1^{\text {a Série }}$ & $7(13,2 \%)$ & $5(13,5 \%)$ \\
$2^{\text {a Série }}$ & $10(18,9 \%)$ & $8(21,6 \%)$ \\
$3^{\text {a Série }}$ & $6(12,2 \%)$ & $8(21,6 \%)$ \\
$4^{\text {a Série }}$ & $12(24,5 \%)$ & $5(13,5 \%)$ \\
$5^{\text {a Série }}$ & $9(18,4 \%)$ & $4(10,8 \%)$ \\
$6^{\text {a Série }}$ & $5(10,2 \%)$ & $3(8,1 \%)$ \\
Total & $53(54,6 \%)$ & $37(38,1 \%)$ \\
\hline
\end{tabular}

\section{Instrumentos}

Para o estudo foram utilizados os seguintes instrumentos:

1. Questionário de dados sócio-demográficos, respondido pela criança.

2. Matrizes Coloridas Progressivas de Raven, Escala Especial (Angelini et al., 1999). Este teste avalia raciocínio analógico e é dividido em três séries A (apreensão da identidade e mudança em padrões contínuos), $\mathrm{Ab}$ (apreensão de figuras distintas com todos espacialmente relacionados) e B (apreensão de mudanças análogas em figuras relacionadas espacialmente e logicamente). Em cada uma destas a criança é solicitada a visualizar uma figura incompleta e identificar, dentre seis alternativas, qual aquela que completaria adequadamente o desenho.

3. Escala de Avaliação de Desempenho Escolar respondida pelas professoras, cujo objetivo é confirmar a ausência de comportamentos indicativos de problemas educacionais das crianças. Este instrumento é composto por 28 perguntas no formato likert de cinco pontos, sendo que um escore alto significa um bom desempenho em relação à aprendizagem e ao comportamento. Essa escala já foi utilizada em outros estudos (Bandeira \& Hutz, 1994) com propósitos semelhantes aos da presente pesquisa.

4. Desenho da Figura Humana, aplicado conforme a proposta de Wechsler (1996/2003), na qual são solicitados um desenho de uma pessoa e um desenho do sexo oposto. O desenho é pontuado conforme o número de itens desenhados pela criança (por exemplo, cabeça, braços, pernas, etc.). 


\section{Procedimentos}

Para a coleta dos dados, inicialmente foi realizado o contato com duas escolas, as quais foi solicitada a lista de crianças com idades entre 6 e 12 anos. A partir da mesma, foi realizado um sorteio das crianças com idade, escolaridade e sexo equivalentes à amostra clínica, prevista no estudo de Arteche (2006), aprovado pelo Comitê de Ética da Universidade Federal do Rio Grande do Sul (UFRGS), composta por crianças que se encontravam em atendimento psicológico. Essa amostra clínica seria posteriormente comparada ao grupo de controle a fim de avaliar a validade de uma escala de indicadores emocionais do DFH.

Após a seleção das crianças, as professoras foram então solicitadas a preencher uma escala de avaliação de desempenho de cada criança, a fim de descartar da amostra crianças que, embora não recebessem nenhum acompanhamento, teriam indicação para tal. Aquelas que se mantiveram na amostra e cujos responsáveis aceitaram a participação na pesquisa mediante o preenchimento do consentimento informado (12 pais não enviaram o consentimento informado), foram convidadas a preencher o questionário de dados sócio-demográficos. Logo em seguida, foram solicitadas a realizar o Desenho da Figura Humana conforme a proposta de Wechsler (1996/2003) e a responder às Matrizes Progressivas Coloridas de Raven. As aplicações foram realizadas de forma individual nas crianças até oito anos e em pequenos grupos para as crianças maiores. A partir desses desenhos, foi feita a análise do $\mathrm{DFH}$, por dois juízes cegos treinados para a proposta de Wechsler.

\section{Análise dos Dados}

Após o levantamento dos itens da escala Wechsler, os dados foram inseridos no software SPSS. Foram contabilizados os totais brutos de cada instrumento. No caso do DFH, foram contabilizados os totais para as figuras masculina e feminina de cada criança, assim como a soma das duas figuras. Para informações sobre os índices de fidedignidade do DFH, foram calculados os índices de concordância entre os juízes e as correlações entre os desenhos masculino e feminino das crianças. A fim de investigar a validade convergente, foi realizada uma análise de correlação de Pearson do DFH com o resultado do teste Raven. Para validade concorrente, a correlação foi realizada com o total da escala de desempenho.

\section{Resultados e Discussão}

Foram calculadas as médias na escala de desempenho, no Raven e no DFH por sexo a fim de verificar se havia diferença significativa entre eles, utilizando-se a Análise de Covariância controlada por idade. Na escala de desempenho escolar, que varia de 28 a 140, os meninos obtiveram média de 96,4 $(D P=22,57)$ e as meninas, 114,9 $(D P=17,81)$. Esse foi o único teste cuja análise estatística mostrou diferença significativa entre as médias $(F(1$, 84) $=15,32, p<0,001)$.

No teste Raven, os meninos atingiram média de 27,1 $(D P=5,87)$ e as meninas, 25,1 $(D P=7,05)$, diferença não significativa ( $p=0,209)$. Analisando-se os resultados por faixa etária a fim de poder estabelecer comparações do desempenho das crianças com as normas apresentadas nos manuais, foram encontradas médias relativamente mais elevadas (em torno de três pontos, em média) do que as apresentadas por Bandeira et al. (2004) na cidade de Porto Alegre, RS (ver Tabela 2). Provavelmente, os valores mais elevados nas médias encontradas neste estudo estejam relacionados com o fato da amostra ter sido indicada, por avaliação dos professores, como não apresentando indicação para tratamento psicológico ou pedagógico.

\section{Tabela 2}

Médias (DP) por Faixa Etária nos Testes DFH e Raven

\begin{tabular}{lll}
\hline Faixa etária $(\mathrm{n})$ & DFH & Raven \\
\hline $6(11)$ & $41,4(12,1)$ & $18(4,1)$ \\
$7(8)$ & $58,6(11,8)$ & $23,1(4,5)$ \\
$8(16)$ & $54,3(12,8)$ & $23,2(6,5)$ \\
$9(19)$ & $68,0(10,2)$ & $29,5(4,1)$ \\
$10(16)$ & $65,9(7,6)$ & $29,0(4,4)$ \\
$11(11)$ & $63,9(11,1)$ & $26,4(5,7)$ \\
$12(9)$ & $72,9(15,6)$ & $32,8(3,7)$ \\
Total $(90)$ & $61,1(14,4)$ & $26,3(6,4)$ \\
\hline
\end{tabular}

Quanto ao DFH, foram realizadas análises de concordância entre juízes por item avaliado. Esses índices ficaram em torno de $91,5 \%$ a 100,0\%. Análises de consistência interna (Kuder-Richardson) indicaram índices ótimos: 0,88 para o primeiro desenho e 0,87 para o segundo desenho, similares aos encontrados por Wechsler (1996/2003). Um índice elevado de correlação foi encontrado entre $o$ desenho feminino e o masculino $(r=0,74, p<0,001)$. Esses resultados indicam bons níveis de fidedignidade do DFH nessa amostra.

Ainda no DFH, os meninos obtiveram média de 60,7 $(D P=15,02)$ e as meninas, $61,7(D P=13,72)$, médias similares apenas às encontradas por Wechsler (1996/2003) na cidade de Ribeirão Preto, SP, dentre estudos em várias outras cidades. Nos outros estudos, as médias são em geral mais baixas. Mais uma vez, o fato de estarmos com uma amostra mais selecionada deve ter interferido nesses resultados. Essa diferença também não foi significativa $(p=0,386)$. As médias por faixa etária também são apresentadas na Tabela 2.

A fim de cumprir os propósitos deste artigo, foram realizadas correlações de Pearson entre os três instrumentos utilizados (Tabela 3). Em ambos os testes (DFH e Raven), não foram encontradas diferenças significativas entre os sexos, corroborando estudos anteriores que indicam que meninos e meninas possuem escores similares em avaliações de inteligência (Duckworth \& Seligman, 2006). Por isso, as análises foram todas realizadas considerando a amostra total. No caso da Escala de Desempenho Escolar, as correlações foram controladas por sexo e idade já que as 
análises apresentaram diferença entre os sexos e a variação nessa escala não está relacionada com a idade, diferentemente do DFH e do Raven.

Tabela 3

Correlação entre os Testes Raven, DFH e a Escala de Desempenho Escolar

\begin{tabular}{lll}
\hline & DFH & Raven \\
\hline Escala & $0,34^{*}$ & $0,42^{*}$ \\
DFH & - & $0,50 *$ \\
\hline
\end{tabular}

Nota. ${ }^{*} p<0,01$.

Os índices mostram evidências de validade do DFH Sistema Wechsler. A correlação entre os dois instrumentos está exemplificada pela Figura 1, na qual é possível verificar que as médias dos dois testes variam simultaneamente. Esse nível de correlação é maior que o encontrado por Wechsler e Schelini (2002), em seu estudo de validade com o TNVRI. Um dos motivos para essa diferença pode ser o fato de ter sido utilizado um instrumento como o Raven, um instrumento tradicional de avaliação cognitiva, com muitos estudos de validação.

Médias no DFH e no Raven por faixa etária

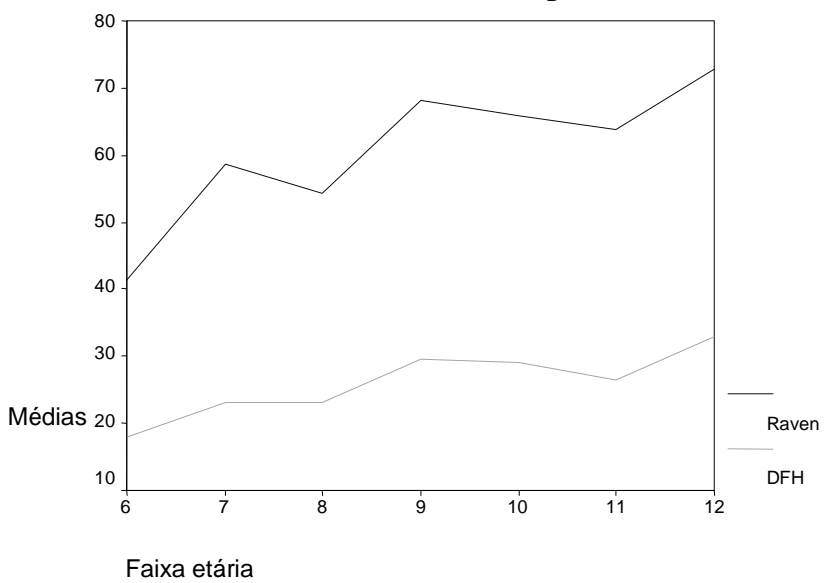

Figura 1. Médias no DFH e no Raven por faixa etária

Pode-se pensar que o construto avaliado pelo DFH aproxime-se mais daquele avaliado pelo Raven do que da faceta da inteligência avaliada através do TNVRI. Embora tanto o Raven quanto o TNVRI proponham-se a avaliar raciocinio abstrato, as sub faces dos instrumentos são diferentes, tendo o TNVRI um foco maior no raciocínio analógico concreto do que o Raven. Reforça-se assim a existência da relação entre o desenvolvimento cognitivo infantil, quando medido pelo desenho e o raciocínio analógico, de forma abstrata. Esses resultados apóiam os estudos de Abell, Horkheimer e Nguyen (1998), Abell, Wood e Liebman (2001) e Marín Rueda e Sisto (2006), sugerindo também que o DFH possa ser incluído em baterias de avaliação de crianças ou em triagens para futuras avaliações mais complexas.
A presente pesquisa contribui, assim, para a confirmação do caráter diagnóstico do DFH. No entanto, fazem-se necessários novos estudos de validade concorrente buscando examinar as correlações do DFH com medidas de inteligência cristalizada, assim como com medidas de desempenho acadêmico. Desta forma, poder-se-á melhor compreender o construto avaliado por esta técnica, bem como o caráter preditivo da mesma.

Além disso, conclui-se que o teste do DFH - Sistema Wechsler apresenta evidências de validade e fidedignidade para a população de Porto Alegre. Sugere-se que estudos com uma amostra maior possam ser conduzidos de forma a gerar mais evidências de validade desse instrumento para a nossa população.

\section{Referências}

Abell, S., Horkheimer, R., \& Nguyen, S. (1998). Intellectual evaluations of adolescents via Human Figure Drawings: An empirical comparison of two methods. Journal of Clinical Psychology, 54(6), 811-815.

Abell, S., Wood, W., \& Liebman, S. (2001). Children's Human Figure Drawings as measures of intelligence: The comparative validity of three scoring systems. Journal of Psychoeducational Assessment, 19(3), 204-215.

Abou-Jamra, C., \& Castillo, M. (1987). Teste de Matrizes Progressivas de Raven. In M. Ancona-Lopez (Ed.), Avaliação da inteligência: Vol. 2. São Paulo, SP: EPU.

Alves, I. (1981). O Teste Goodenough-Harris em pré-escolares paulistanos. Boletim de Psicologia, 80(33), 40-52.

Angelini, A. L., Alves, I. C. B., Custódio, E. M., Duarte, W. F., \& Duarte, J. L. M. (1999). Matrizes Progressivas Coloridas de Raven: Escala especial. Manual. São Paulo, SP: Centro Editor de Testes e Pesquisa em Psicologia.

Arteche, A. X. (2006). Indicadores emocionais do desenho da figura humana: Construção e validação de uma escala infantil. Tese de Doutorado não-publicada, Universidade Federal do Rio Grande do Sul, Porto Alegre, RS.

Bandeira, D. R., Alves, I. B., Giacomel, A., \& Lorenzatto, L. (2004) Matrizes Progressivas Coloridas de Raven - Escala especial: Normas para Porto Alegre, RS. Psicologia em Estudo (Maringá), 9(3), 497-486.

Bandeira, D., \& Hutz, C. (1994). A contribuição dos testes DFH, Bender e Raven na predição do rendimento escolar na primeira série. Psicologia: Teoria e Pesquisa, 10(1), 59-72.

Conselho Federal de Psicologia. (2003). Resolução CFP $n^{o}$ 02/ 2003. Brasília, DF.

Cox, M. (1995). Desenho da criança. São Paulo, SP: Martins Fontes.

Costenbader, V., \& Ngari, S. M. (2001). A Kenya Standardization of the Raven's Coloured Progressive Matrices. School Psychology International, 22(3), 258-268.

Duckworth, A., \& Seligman, M. (2006). Self-discipline fives girls the edge: Gender in self-discipline, grades and achievement test scores. Journal of Educational Psychology, 98(1), 198208.

Goodenough, F. (1974). Test de inteligência infantil por medio del dibujo de la figura humana (7. ed.). Buenos Aires, Argentina: Paidós.

Kamphaus, R., \& Pleiss, K. (1991). Draw-a-Person techniques: Tests in search of a construct. Journal of School Psychology, 29, 395-491. 
Koppitz, E. M. (1984). El dibujo de la figura humana em los niños. Buenos Aires, Argentina: Guadalupe.

Marín Rueda F. J., \& Sisto F., (2006). Estudo sobre as categorias de interpretação das Matrizes Coloridas de Raven e DFHEscala Sisto. Aletheia, 23, 17-26.

Naglieri, J. (1988). DAP: Draw a person: A quantitative scoring system. San Diego, CA: The Psychological Corporation.

Parvathi, S., \& Natarajan, P. (1985). A study of the drawing abilities of children as related to abstract intelligence. Journal of Indian Academy of Applied Psychology, 11(2), 21-24

Pasquali, L. (2001). Técnicas de exame psicológico - TEP: Manual. São Paulo, SP: Casa do Psicólogo.

Pasquali, L. (2005). Manual técnico e de aplicação do Teste Não-Verbal de Raciocínio para Crianças - TNVRI. São Paulo, SP: Vetor.

Pind, J., Gunnarsdóttir, E. K., \& Jóhannesson, H. S. (2003). Raven's Standard Progressive Matrices: New school age norms and a study of the test's validity. Personality and Individual Differences, 34(3), 375-386.

Powers, S., \& Brakan, J. (1986). Concurrent validity of the Standard Progressive Matrices for Hispanic and non Hispanic seventh-grade students. Psychology in the Schools, 23(4), 333336.

Sisto, F. (2006). O desenho da figura humana - Escala Sisto. São Paulo, SP: Vetor.

Wechsler, S. (2003). DFH III: O desenho da figura humana: Avaliação do desenvolvimento cognitivo de crianças brasileiras. Campinas, SP: Editora da Pontifícia Universidade Católica de São Paulo. (Original publicado em 1996)

Wechsler, S., \& Schelini, P. (2002). Validade do desenho da figura humana para avaliação cognitiva infantil. Avaliação Psicológica, 1(1), 29-38. 